\title{
Simulação clínica no atendimento de enfermagem à mulher no terceiro trimestre gestacional: validação de cenário
}

\author{
Clinical simulation of nursing care to women in the third trimester of pregnancy: validation of a \\ scenario
}

\section{Simulación clínica en la atención de enfermería a mujeres en el tercer trimestre gestacional: validación del escenario}

\section{RESUMO}

Objetivo: Elaborar e validar um cenário para simulação clínica e checklist para avaliação do ensino sobre consulta de enfermagem no terceiro trimestre gestacional. Método: Estudo metodológico em 5 etapas (Overview, Scenario, Scenario Desing Progression, Debriefing e Assessment), desenvolvido de novembro de 2019 a julho de 2020. A validação foi realizada pelos juízes de forma remota, com preenchimento de uma escala likert. Foi calculado o Índice de Validade de Conteúdo (IVC). Resultados: O cenário "Conduta do enfermeiro frente a uma gestante em fase latente do trabalho de parto na consulta de pré-natal" e seu checklist foram validados por 5 juízes, com IVC igual a 1,0. Conclusão: O processo de validação atesta o rigor científico com o qual o cenário e o ckecklist foram elaborados, assim permite que seja utilizado com segurança, no ensino de graduação em enfermagem. Espera-se que este estudo estimule novas pesquisas sobre a temática.

Descritores: Treinamento por Simulação; Educação em Enfermagem; Estudo de Validação; Cuidado Pré-Natal; Início do Trabalho de Parto.

\begin{abstract}
Objective: To develop and validate a clinical simulation scenario and a checklist to evaluate teaching/learning aspects about nursing consultation to pregnant women in the third gestational trimester. Method: Methodological study in 5 stages (Overview, Scenario, Scenario Desing Progression, Debriefing and Assessment), developed from November 2019 to July 2020. The validation was performed remotely by the judges, with the completion of a Likert scale. The Content Validity Index (CVI) was calculated. Results: The scenario "Nurses' actions before a pregnant woman in the latent phase of labor during the prenatal consultation" and its checklist were validated by 5 judges, reaching a CVI equal to 1.0. Conclusion: The validation process attests to the scientific rigor with which the scenario and ckecklist were elaborated and can, therefore, be used safely in undergraduate nursing education. It is hoped that this study will stimulate further research on the subject.

Descriptors: Simulation Training; Education, Nursing; Validation Study; Prenatal Care; Labor Onset.
\end{abstract}

\section{RESUMEN}

Objetivo: Desarrollar y validar un escenario para la simulación clínica y de un checklist para la evaluación de la enseñanza y el aprendizaje de una consulta de enfermería en el tercer trimestre del embarazo. Método: Estudio metodológico en 5 etapas (Overview, Scenario, Scenario Desing Progression, Debriefing e Assessment) desarrollado de noviembre de 2019 a julio de 2020. La validación fue realizada remotamente por los evaluadores, con la cumplimentación de una escala Likert. Se calculó el Índice de Validez de Contenido (IVC). Resultados: El escenario "Conducta de la enfermera hacia una mujer embarazada latente en el cuidado prenatal" y su checklist fueron validados por cinco evaluadores con un IVC de 1.0. Conclusión: La validación da fe del rigor científico con el que se desarrollaron el escenario y el checklist, lo que permite utilizarlo con seguridad en la educación en enfermería. Se espera que este estudio estimule más investigaciones sobre el tema.

Descriptores: Entrenamiento Simulado; Educación en Enfermería; Estudio de Validación; Atención Prenatal; Inicio del Trabajo de Parto.
Rafaela Gomes Portela ${ }^{1}$

0000-0001-6822-0366

Alecssandra de Fátima Silva

Viduedo ${ }^{1}$

0000-0002-3529-3814

Laiane Medeiros Ribeiro ${ }^{1}$

0000-0002-5041-8283

Casandra Genoveva Rosales

Martins Ponce de Leon ${ }^{1}$

0000-0003-4378-9200

Juliana Machado Schardosim ${ }^{1}$

(D) 0000-0003-2368-5834

${ }^{1}$ Universidade de Brasília.

Autor correspondente:

Rafaela Gomes Portela

E-mail: rafaelagomez.p25@gmail.com

\section{Como citar este artigo:}

Portela RG, Viduedo AFS, Ribeiro LM, et al. Simulação clínica no atendimento de enfermagem à mulher no terceiro trimestre gestacional: validação de cenário. Revista de Enfermagem do Centro-Oeste Mineiro. 2021;11:e4123. [Access___ ] Available in:___. DOI: http://doi.org/10.19175/recom.v10i0.4 123 


\section{INTRODUÇÃO}

Cada fase da gestação possui singularidades que exigem do enfermeiro abordagens e cuidados específicos. Por isso, nas consultas de pré-natal do terceiro trimestre, em razão da proximidade do parto e à ansiedade que ele gera na gestante e em sua família, é importante que o enfermeiro esteja apto para oferecer orientações precisas, visando z tranquilizá-los e z prepará-los para um parto seguro e informado; demostrar conhecimentos e habilidades técnicas para realizar a avaliação clínica e obstétrica; e reconhecer o trabalho de parto e suas fases, sendo resolutivo nas situações identificadas ${ }^{(1-2)}$.

O reconhecimento do início do trabalho de parto é crucial para que as parturientes sejam conduzidas à unidade de saúde, no momento correto, evitando a internação precoce que pode culminar em um trabalho de parto mais longo e sofrido para a gestante, bebê e acompanhante; excesso de intervenções ou, ainda, em cesarianas desnecessárias.

A Organização Mundial de Saúde (OMS) divide, didaticamente, o parto em quatro fases, também denominadas de períodos clínicos. O primeiro período é o trabalho de parto, que se subdivide em fase latente, marcada por contrações que podem ser dolorosas, com padrão regular e progressão mais lenta, que repercutem em apagamento do colo uterino e dilatação cervical de até $5 \mathrm{~cm}$; fase ativa, que caracteriza-se por contrações dolorosas e regulares com apagamento do colo mais rápido de $5 \mathrm{~cm}$ até à dilatação completa e a fase de transição que caracteriza-se pelo início da fase de expulsão. O segundo período é o expulsivo, que corresponde ao parto propriamente dito. $O$ terceiro período corresponde à dequitação placentária e o quarto período às primeiras horas de recuperação da puérpera. A duração de cada fase é singular em cada parturiente, entretanto essa fase não costuma ultrapassar 8 horas de duração ${ }^{(3)}$.

No intuito de atender às novas demandas do setor saúde e acompanhar a crescente valorização do papel da enfermagem na Atenção Primária à Saúde (APS) para o cuidado pré-natal, é necessário estimular o uso de metodologias inovadoras de ensino-aprendizagem, disponibilizar recursos adequados para o ensino e associar a teoria à prática ${ }^{(4)}$. Nesse sentido, a simulação clínica é um tipo de metodologia ativa de ensino que combina prática com teoria e incentiva o aluno a participar da construção ou fixação de seus conhecimentos, por meio da vivência de cenários que buscam imitar a realidade.

Os cenários simulados possibilitam que os alunos desenvolvam e exercitem habilidades técnicas e não técnicas para um cuidado focado na segurança do paciente e adquiram maior confiança e segurança, caso vivenciem essas mesmas situações no futuro, durante sua prática clínica ${ }^{(5-6)}$. No entanto, para que os objetivos de aprendizagem propostos para cada cenário sejam atingidos, é fundamental incluir, ao fim da simulação, o debriefing, pois essa etapa estimula a reflexão sobre a experiência simulada, permitindo o aprimoramento da aprendizagem e o aumento da autoconsciência e autoeficácia dos participantes $^{(7)}$.

A elaboração de cenários clínicos é complexa, em decorrência dos detalhes que necessitam ser planejados, visando a uma maior similaridade da simulação clínica à realidade dos contextos da prática nos ambientes de saúde. Nesse sentido, a construção de um caso clínico pode se distinguir em três modalidades (alta, média e baixa fidelidade), de acordo com os níveis de proximidade com a realidade que se deseja reproduzir e o objetivo da aprendizagem que se busca alcançar ${ }^{(8-9)}$. Além disso, é recomendado que, após a construção do cenário, ele seja validado por juízes/experts e/ou público-alvo o que garante a sua segurança técnica e integridade para ser, posteriormente, reproduzido ${ }^{(10-11)}$.

Ainda que a simulação seja, comprovadamente, uma estratégia capaz de agregar valor ao ensino de enfermagem ${ }^{(12)}$, não foram encontrados, até o presente momento, na literatura nacional e internacional, estudos relativos à elaboração e validação de cenário de simulação clínica sobre a assistência de enfermagem às consultas de terceiro trimestre do pré-natal de risco habitual. Nesse sentido, o Brasil, em 2007, criou o Pró-saúde, um programa do Ministério da Saúde que incentiva o elo entre a formação de graduação e a necessidade da população, tendo como eixo central a integração ensino-serviço, por meio da vivência dos estudantes, no cenário real de práticas da APS ${ }^{(4)}$, uma vez que a vivência do pré-natal é considerada primordial a fim de alcançar um desfecho positivo, na saúde da futura mãe e sua família, no ciclo gravídico puerperal. Diante disso, neste estudo, objetivou-se elaborar e validar um cenário para a simulação clínica e um checklist para avaliação do ensino/aprendizado sobre a consulta de 
enfermagem, no terceiro trimestre gestacional para o ensino de graduação em enfermagem.

\section{MÉTODO}

Trata-se de um estudo do tipo metodológico, que adotou os pressupostos de Gilbert e Adamson (2016) ${ }^{(13)}$ a partir de recomendações da International Nursing Association for Clinical Simulation and Learning (INACSL), para elaboração e validação de um cenário de simulação clínica. De acordo com o referencial metodológico adotado, foram seguidas as 5 etapas:

Overview: reflexão sobre questões que embasaram a elaboração do cenário, tais como: necessidades organizacionais, do educador e de aprendizagem dos alunos, por exemplo, as habilidades e/ou competências a serem trabalhadas na simulação relacionadas à consulta de pré-natal.

Scenario: elaboração do cenário, a partir de uma base teórica sólida, caso clínico relevante e ambiente próximo à realidade da consulta de terceiro trimestre de risco habitual.

Scenario Design Progression: Definição de papéis/ atores, scripts e decisões sobre uso de manequim/simulador e sua configuração.

Debriefing: Elaboração de um checklist para auxiliar o processo avaliativo docente e a condução do debriefing na atividade simulada, com pontos de discussão específicos para o cenário validado.

Assessment: Avaliação pelos juízes sobre os documentos produzidos, ao longo das etapas anteriores.

Para que a etapa de validação do cenário e do checklist fosse viabilizada, em meio à pandemia do coronavírus, optou-se pela validação de forma online. Desse modo, foram selecionados e convidados 11 juízes com expertise na temática abordada no cenário, a partir das informações contidas na Plataforma Lattes e disponibilidade de e-mail para envio do convite. Apenas cinco juízes responderam.

Os critérios de inclusão foram: profissionais com experiência em saúde da mulher que obtiveram, no mínimo, 4 pontos no Sistema de Pontuação para a Seleção de Juízes adaptado a partir de Góes et al. (2014) $)^{(14)}$, considerando titulação, experiência profissional, área de ensino, desenvolvimento/ orientação de pesquisas e publicações. Para cálculo da pontuação, foram consultados os currículos da Plataforma Lattes dos profissionais. Os critérios de exclusão foram: profissionais que estavam afastados de atividades assistenciais ou de ensino, por dois anos ou mais, por se entender que talvez não houvesse conhecimento atualizado sobre as evidências científicas.

As quatro primeiras etapas foram de estudo teórico, e ocorreram apenas com a participação das pesquisadoras. Já, a quinta etapa, consistiu na validação em si. Os especialistas foram contactados por e-mail convite contendo: um breve resumo do estudo, Termo de Consentimento Livre e Esclarecido (TCLE) assinado pelas pesquisadoras, arquivo em $\mathrm{pdf}_{\bar{T}}$ contendo a descrição completa do cenário, o checklist proposto para a avaliação do ensino e aprendizado, ficha de caracterização dos juízes e um link de acesso ao formulário elaborado na plataforma Google Forms ${ }^{\circledR}$, para preenchimento, após a leitura dos documentos, sobre a avaliação acerca do cenário e checklist elaborados. O estudo foi desenvolvido de novembro de 2019 a julho de 2020, sendo a etapa de validação online realizada entre maio e julho de 2020.

O formulário do Google Forms ${ }^{\circledR}$ incluiu uma escala likert, avaliando 18 aspectos referentes ao cenário e checklist, para avaliação do ensino e aprendizado. Cada aspecto avaliado possuía 4 alternativas de respostas: totalmente inadequado; inadequado, mas pode ser reformulado; adequado com pequenos ajustes e totalmente adequado. Os aspectos avaliados incluíram questões técnicas sobre o cenário, questões relacionadas ao ensino como apropriação ao nível de conhecimento dos alunos, sobre o realismo do cenário, sobre a árvore de tomada de decisões e questões relacionadas ao debriefing.

Após a obtenção dos dados, procedeu-se ao cálculo do Índice de Validade de Conteúdo (IVC), no software Microsoft Excel ${ }^{\circledR}$, versão 2016. Para a análise do IVC obtido, considerando-se o quantitativo de juízes inferior a 6 , estipulou-se um IVC mínimo de 1,0 entre os juízes( ${ }^{(15)}$. As duas alternativas denominadas como "adequado" pontuaram, positivamente, no cálculo do IVC. Dados relacionados ao perfil dos juízes foram analisados por estatística descritiva e expressos em frequências absoluta e relativa.

O estudo foi aprovado pelo Comitê de Ética da instituição de origem sob CAAE no 03107418.5.0000.8093 e os pressupostos das resoluções 466/12 e 510/2016, do Conselho Nacional de Saúde, foram rigorosamente seguidos ${ }^{(16)}$. 


\section{RESULTADOS}

O cenário elaborado intitulado "Conduta do enfermeiro frente a uma gestante em fase latente do trabalho de parto na consulta de pré-natal" versava sobre uma parturiente nulípara com 35 anos de idade e com 38 semanas e 2 dias de idade gestacional que foi, juntamente com seu acompanhante, a uma consulta rotineira de prénatal do terceiro trimestre em uma Unidade Básica de Saúde (UBS). Na consulta, ela referiu cólicas fortes, mas suportáveis. Até o momento da consulta, não havia ocorrido perda de líquido amniótico nem sangramento vaginal. O cenário propõe que os estudantes que interpretarão os enfermeiros, deverão identificar que a parturiente está na fase latente de trabalho de parto, apresentando habilidades técnicas e conhecimento para a tomada de decisão, nessa fase do trabalho de parto na Atenção Básica. É importante frisar que, antes da realização da simulação desse cenário, o educador deverá trabalhar em sala de aula os conteúdos teóricos sobre assistência pré-natal, embriologia e fisiologia da gestação e do trabalho de parto para que os alunos possam atingir os objetivos de aprendizagem propostos.

Para que o ambiente seja o mais realista possível, é recomendado que o cenário possua todos os materiais compatíveis com um consultório de Unidade Básica de Saúde: Mobiliário/ Decoração - mesa, 4 cadeiras, maca, escadinha, lixeira, calendário de mesa, cartazes e impressos sobre campanhas do Ministério da Saúde que, normalmente, se encontram nos consultórios das UBSs; Materiais médicohospitalares - Sonar Obstétrico e reprodução de som de ausculta real de Batimento Cardíaco Fetal (BCF) "real", esfigmomanômetro, estetoscópio, fita métrica, termômetro, lanterna clínica, frasco de gel para o sonar, álcool 70\%, luvas estéreis, camisola hospitalar, lençol descartável para maca, lençol extra para cobrir paciente em eventual exame de toque, folhas em branco e canetas; Documentos - caderneta da gestante preenchida com as consultas anteriores, plano de parto, bloco de receituário, bloco de formulário de solicitação de exames, bloco de relatório de encaminhamento da gestante para a maternidade. Na Figura 1, apresenta-se o cenário elaborado de forma resumida.

Figura 1 - Resumo do cenário intitulado "Conduta do enfermeiro frente a uma gestante em fase latente do trabalho de parto na consulta de pré-natal", Brasília, 2020.

\begin{tabular}{|l|l|}
\hline $\begin{array}{l}\text { Objetivos } \\
\text { aprendizagem }\end{array}$ & $\begin{array}{l}\text { Realizar a consulta de enfermagem no pré-natal de risco habitual, identificando o trabalho de parto em fase } \\
\text { latente e dar os encaminhamentos e orientações corretas }\end{array}$ \\
\hline Complexidade & Média \\
\hline Fidelidade & Média \\
\hline Duração & Até 20 minutos \\
\hline Participantes & $\begin{array}{l}\text { 2 alunos para interpretar os enfermeiros } \\
\text { 2 atores para interpretar a gestante e seu acompanhante }\end{array}$ \\
\hline Figurino & $\begin{array}{l}\text { Gestante: Roupas comuns para pessoas na faixa etária próxima de 35 anos e uso de modelador para proteger } \\
\text { exposição de partes íntimas da atriz e acomodar as peças anatômicas de silicone adaptadas ao figurino } \\
\text { (mamas e barriga de gestante). } \\
\text { Acompanhante: A definição do sexo e parentesco do acompanhante ficará a cargo de cada instituição que } \\
\text { adotar este cenário no ensino. Portanto, a definição de figurino e maquiagem também. }\end{array}$ \\
\hline Caso clínico sucinto & $\begin{array}{l}\text { A.P.L, 35 anos, chega acompanhada à UBS para uma consulta de rotina de pré-natal do terceiro trimestre } \\
\text { referindo cólicas fortes, mas suportáveis. Data da Última Menstruação: deverá ser fornecida ao aluno, de } \\
\text { modo que a gestante esteja com 38 semanas e 2 dias de idade gestacional, no dia da simulação. Sinais vitais } \\
\text { e medidas antropométricas: Frequência Cardíaca: 85bpm; Pressão Arterial: } 130 \text { x 80mmH; Frequência } \\
\text { Respiratória: 18irpm; Saturação: 98\%; Peso: 75kg; Altura: 1,60m. } \\
\text { Observação: A atriz possui os seguintes dispositivos: prótese de mamas de silicone e barriga compatível com } \\
\text { o período gestacional e simulador pélvico feminino para treinamento clínico avançado. }\end{array}$ \\
\hline \multicolumn{1}{|c|}{ Fonte: Elaborado pelas pesquisadoras a partir de dados obtidos do estudo. }
\end{tabular}

De acordo com a INACSL, é fundamental utilizar métodos facilitadores antes, durante e após a simulação, com esse intuito, foi elaborada uma árvore de tomada de decisões, representada pela Figura 2, visando z alcançar os objetivos da aprendizagem e a progressão esperada para o caso clínico ${ }^{(17)}$. Nesse instrumento, estão contidas dicas que os atores podem fornecer aos alunos, durante a simulação, caso sejam necessárias, para que eles atinjam os objetivos de aprendizagem. 
Figura 2- Árvore de tomada de decisões. Brasília, 2020.

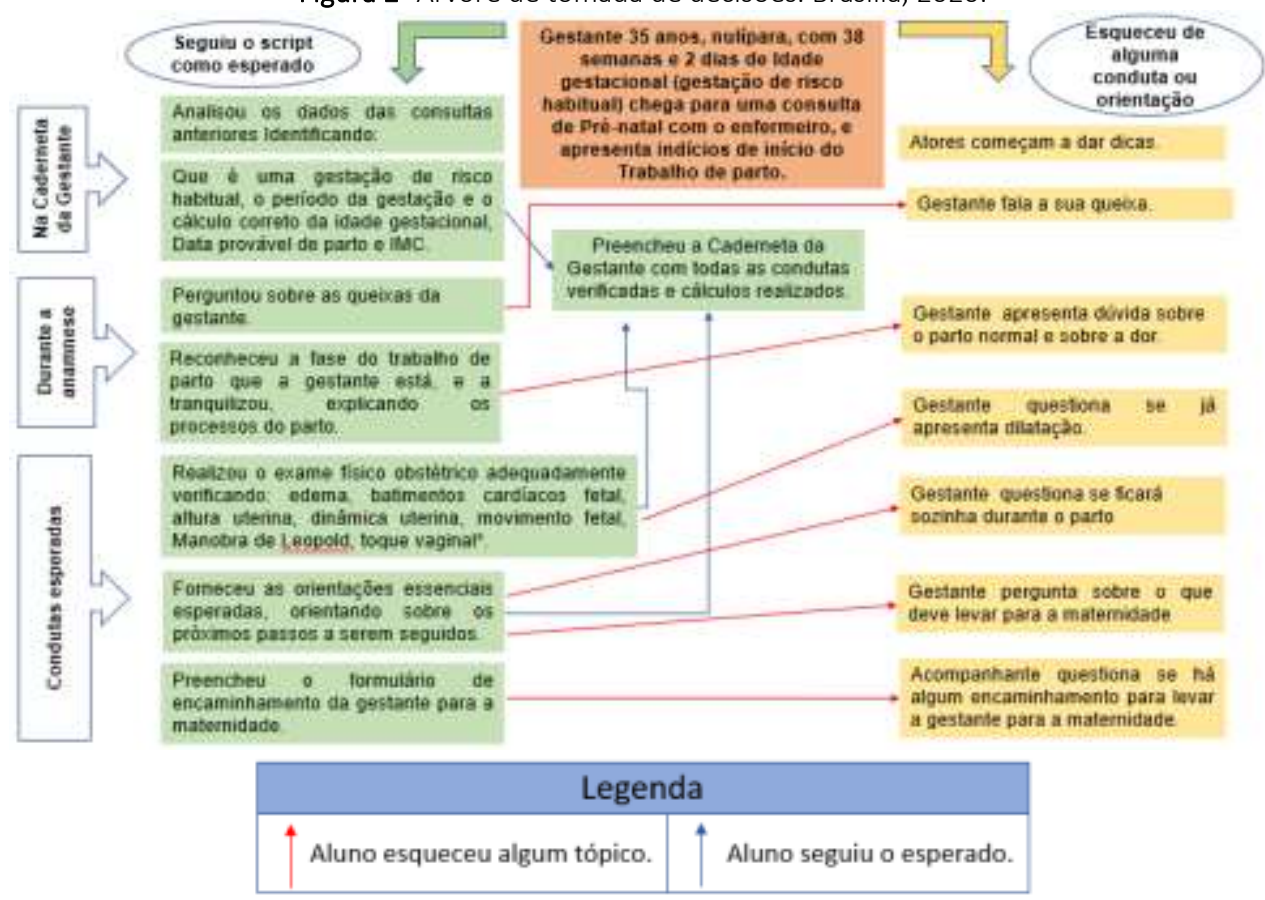

Fonte: Elaborado pelas pesquisadoras a partir de dados obtidos do estudo.

O checklist, para avaliação do ensino e aprendizado validado, foi estruturado em 4 tópicos: postura, anamnese, condutas e orientações essenciais. Na postura, são avaliados aspectos relacionados ao acolhimento, comunicação, relacionamento interpessoal e segurança, durante $\mathrm{o}$ atendimento. Na anamnese, são avaliados aspectos referentes à qualidade da entrevista realizada junto à paciente e acompanhante na consulta. Nas condutas, são avaliadas as habilidades técnicas e o raciocínio clínico dos estudantes frente ao caso, para correto encaminhamento da situação. Nas orientações essenciais são avaliadas as orientações fornecidas pelos alunos à parturiente e acompanhante sobre a fase do trabalho de parto em que se encontra e sobre tudo que se considera importante para esse caso. Esse checklist foi proposto para padronizar a condução do debriefing desse cenário, validado.

Figura 3 - Checklist de Habilidades e Conhecimentos Esperados dos Alunos para Condução do Debriefing. Brasília, 2020.

\begin{tabular}{|c|c|c|c|c|}
\hline \multicolumn{5}{|l|}{ Checklist de Habilidades e Conhecimentos Esperados dos Alunos } \\
\hline & NR & 1 & PA & A \\
\hline \multicolumn{5}{|l|}{ POSTURA } \\
\hline \multicolumn{5}{|l|}{$\begin{array}{l}\text { Apresentaram-se e acolheram a mulher e o acompanhante de maneira respeitosa e } \\
\text { humanizada. }\end{array}$} \\
\hline \multicolumn{5}{|l|}{ Promoveram escuta qualificada e comunicação efetiva durante a consulta. } \\
\hline \multicolumn{5}{|l|}{$\begin{array}{l}\text { Apresentaram bom relacionamento interpessoal com a equipe e com paciente e } \\
\text { acompanhante. }\end{array}$} \\
\hline \multicolumn{5}{|l|}{$\begin{array}{l}\text { Apresentaram capacidade de liderança e segurança na abordagem à gestante e } \\
\text { acompanhante. }\end{array}$} \\
\hline \multicolumn{5}{|l|}{ ANAMNESE } \\
\hline \multicolumn{5}{|l|}{$\begin{array}{l}\text { Realizaram a anamnese, por meio da confirmação de informações já contidas na caderneta } \\
\text { da gestante. }\end{array}$} \\
\hline \multicolumn{5}{|l|}{$\begin{array}{l}\text { Observaram os dados preenchidos sobre consultas anteriores se inteirando sobre a evolução } \\
\text { da gestação atual e Data Provável de Parto. }\end{array}$} \\
\hline \multicolumn{5}{|l|}{ Conferiram os exames já realizados e situação vacinal registrados na caderneta da gestante. } \\
\hline \multicolumn{5}{|l|}{ Perguntaram sobre as queixas e dúvidas da gestante e acompanhante. } \\
\hline \multicolumn{5}{|l|}{ CONDUTAS } \\
\hline $\begin{array}{l}\text { Realizaram cálculo correto do IMC, a partir do peso e altura fornecidos no caso e } \\
\text { preencheram corretamente no gráfico do na caderneta da gestante. }\end{array}$ & & & & \\
\hline
\end{tabular}

(Continua) 
Figura 3 - Checklist de Habilidades e Conhecimentos Esperados dos Alunos para Condução do Debriefing. Brasília, 2020.

\begin{tabular}{|c|c|c|c|c|}
\hline \multicolumn{5}{|l|}{ Checklist de Habilidades e Conhecimentos Esperados dos Alunos } \\
\hline & NR & $\mathrm{I}$ & PA & A \\
\hline \multicolumn{5}{|l|}{ CONDUTAS } \\
\hline \multicolumn{5}{|l|}{$\begin{array}{l}\text { Realizaram cálculo correto da idade gestacional e confirmaram a data provável de parto já } \\
\text { registrada na caderneta da gestante. }\end{array}$} \\
\hline \multicolumn{5}{|l|}{$\begin{array}{l}\text { Conseguiram ser resolutivos com as dúvidas e queixas apresentadas pela gestante e } \\
\text { acompanhante. }\end{array}$} \\
\hline \multicolumn{5}{|l|}{$\begin{array}{l}\text { Realizaram exame obstétrico: a manobra de Leopold, verificando situação e apresentação } \\
\text { fetal, medição da altura uterina, ausculta de batimentos cardíacos fetal, dinâmica uterina e, } \\
\text { se havia movimentos fetais ao toque. Sempre explicando o procedimento e respeitando a } \\
\text { privacidade da mulher. }\end{array}$} \\
\hline \multicolumn{5}{|l|}{$\begin{array}{l}\text { *Verificaram, por meio do exame do toque vaginal se havia dilatação da cérvice uterina, } \\
\text { explicando o procedimento e respeitando a privacidade da mulher. (facultativo) }\end{array}$} \\
\hline \multicolumn{5}{|l|}{ Verificaram a presença de edema em membros inferiores. } \\
\hline \multicolumn{5}{|l|}{ Identificaram que a gestante estava na fase latente do trabalho de parto. } \\
\hline \multicolumn{5}{|l|}{ Tranquilizaram a gestante e acompanhante sobre quando se dirigir a maternidade. } \\
\hline \multicolumn{5}{|l|}{$\begin{array}{l}\text { Preencheram o formulário de referência para encaminhamento da gestante para a } \\
\text { maternidade. }\end{array}$} \\
\hline \multicolumn{5}{|l|}{$\begin{array}{l}\text { Realizaram o registro da consulta na caderneta da gestante, demonstrando conhecimento } \\
\text { sobre a localização dos dados e informações na caderneta da gestante. }\end{array}$} \\
\hline \multicolumn{5}{|l|}{ ORIENTAÇÕES ESSENCIAIS } \\
\hline \multicolumn{5}{|l|}{$\begin{array}{l}\text { Explicaram sobre a evolução fisiológica do trabalho de parto e em qual fase a gestante se } \\
\text { encontrava e sua dilatação da cérvice uterina. }\end{array}$} \\
\hline \multicolumn{5}{|l|}{ Orientaram sobre quando a gestante deveria se dirigir à maternidade. } \\
\hline \multicolumn{5}{|l|}{$\begin{array}{l}\text { Aconselharam sobre os métodos não farmacológicos para o alívio da dor e estímulo à } \\
\text { fisiologia do trabalho de parto. }\end{array}$} \\
\hline \multicolumn{5}{|l|}{$\begin{array}{l}\text { Aconselharam a gestante sobre os benefícios das posições mais verticalizadas, durante o } \\
\text { trabalho de parto. }\end{array}$} \\
\hline \multicolumn{5}{|l|}{ Abordaram os benefícios do parto natural e humanizado. } \\
\hline \multicolumn{5}{|l|}{ Comentaram sobre a Lei do Acompanhante (Lei no11.108/2005). } \\
\hline Orientaram sobre o que a gestante deve levar para a maternidade. & & & & \\
\hline
\end{tabular}

Fonte: Elaborado pelas pesquisadoras a partir de dados obtidos do estudo.

Além do checklist, também foram propostas as seguintes perguntas norteadoras para 0 debriefing: "O que você viu quando entrou no cenário?"; "Como se sentiu durante a simulação?"; "Quais foram os seus pontos positivos?"; "Qual foi a sua reação, após ouvir a apresentação sucinta do caso?"; "A partir do que viu, quais condutas tomou?"; "O que te levou a essa tomada de decisão?"; "Quais eram os objetivos das suas condutas e orientações?"; "Considera que esqueceu de realizar alguma conduta ou orientação importante?"; "Se, fosse reviver essa experiência, faria algo diferente?". Foi sugerido que debriefing tenha duração de até 15 minutos, iniciando pela fala dos alunos, sobre como foi a experiência no cenário simulado e suas impressões sobre aspectos positivos e aspectos a serem melhorados em sua conduta, após esse momento o docente comenta sobre sua avaliação.
Após a construção do caso clínico e elaboração do checklist, realizou-se a validação online do cenário por juízes, experts na área de saúde da mulher e simulação. Dos 11 profissionais convidados, participaram apenas cinco juízas nessa etapa. Das cinco, quatro são docentes, na área de saúde da mulher em Universidades Federais, sendo duas do Centro-Oeste, uma da região Sul e outra da região Nordeste. A quinta juíza é enfermeira assistencial na região Centro-Oeste.

Antes do convite aos juízes, realizou-se uma busca na Plataforma Lattes, para análise dos currículos e cálculo do Sistema de Pontuação de Juízes. Os pontos obtidos pelas juízas foram: 7, 12, 14, 14 e 18 pontos. Na Tabela 1, apresenta-se um perfil das juízas, com dados relacionados a sua formação e atuação profissional e sobre sua experiência com simulação. 
Tabela 1 - Caracterização dos Juízes do Estudo. Brasília, 2020

\begin{tabular}{|c|c|c|}
\hline & $\mathrm{N}$ & $\%$ \\
\hline Idade (anos)* & $48,2 \pm 12,03$ & \\
\hline \multicolumn{3}{|l|}{ Formação } \\
\hline Graduação em Enfermagem & 5 & 100 \\
\hline \multicolumn{3}{|l|}{ Títulos de Pós-graduação } \\
\hline Especialização & 3 & 60 \\
\hline Mestrado & 5 & 100 \\
\hline Doutorado & 3 & 60 \\
\hline \multicolumn{3}{|l|}{ Atuação Profissional } \\
\hline Assistência & 4 & 80 \\
\hline Docência & 5 & 100 \\
\hline Tempo de experiência (anos) ${ }^{\dagger}$ & $21,5(14,5-29,0)$ & \\
\hline \multicolumn{3}{|l|}{ Experiência com Simulação } \\
\hline Docente & 3 & 60 \\
\hline Pesquisador & 1 & 20 \\
\hline Aluno & 1 & 20 \\
\hline Participação como juiz anteriormente & 2 & 40 \\
\hline Tempo de Experiência com Simulação† & $1,5(1,0-2,5)$ & \\
\hline Publicações na área de Saúde da Mulher & 5 & 100 \\
\hline Publicações sobre Simulação Realística & 1 & 20 \\
\hline
\end{tabular}

Fonte: Elaborado pelas pesquisadoras a partir de dados obtidos do estudo.

*Valores expressos em média \pm desvio padrão.

†Valores expressos em mediana e intervalo interquartil.

O cálculo do IVC foi realizado, a partir dos dados obtidos no preenchimento da escala likert, no Google Forms ${ }^{\circledR}$. Na Tabela 2, apresentam-se os 18 critérios avaliados e a pontuação obtida. Nota-se, ao final, que o IVC obtido foi de 1,0, conforme o referencial teórico adotado preconizava ${ }^{(15)}$.

Tabela 2 - Avaliação dos Juízes sobre o Cenário Simulado e Checklist Validados. Brasília, 2020

\begin{tabular}{|c|c|c|c|c|c|c|c|c|c|}
\hline \multirow[t]{2}{*}{ Itens Avaliados } & \multicolumn{2}{|c|}{$\begin{array}{l}\text { Totalmente } \\
\text { Inadequado }\end{array}$} & \multicolumn{2}{|c|}{$\begin{array}{l}\text { Inadequado, } \\
\text { mas pode ser } \\
\text { modificado }\end{array}$} & \multicolumn{2}{|c|}{$\begin{array}{l}\text { Adequado } \\
\text { com } \\
\text { pequenos } \\
\text { ajustes }\end{array}$} & \multicolumn{2}{|c|}{$\begin{array}{l}\text { Totalmente } \\
\text { Adequado }\end{array}$} & \multirow[t]{2}{*}{ IVC } \\
\hline & $n$ & $\%$ & $n$ & $\%$ & $\mathrm{n}$ & $\%$ & $n$ & $\%$ & \\
\hline Plausibilidade do caso clínico & - & - & - & - & 1 & 20 & 4 & 80 & 1,0 \\
\hline Aderência às evidências científicas disponíveis & - & - & - & - & 1 & 20 & 4 & 80 & 1,0 \\
\hline $\begin{array}{l}\text { Construção do cenário de acordo com condutas } \\
\text { preconizadas pelo Ministério da Saúde }\end{array}$ & - & - & - & - & 1 & 20 & 4 & 80 & 1,0 \\
\hline $\begin{array}{l}\text { Adequação do cenário em relação ao apoio didático } \\
\text { fornecido ao aluno }\end{array}$ & - & - & - & - & 1 & 20 & 4 & 80 & 1,0 \\
\hline $\begin{array}{l}\text { Complexidade em relação ao nível de conhecimento e } \\
\text { habilidades do aluno }\end{array}$ & - & - & - & - & 1 & 20 & 4 & 80 & 1,0 \\
\hline Realismo & - & - & - & - & 1 & 20 & 4 & 80 & 1,0 \\
\hline $\begin{array}{l}\text { Descrição sucinta do caso: informações fornecidas ao } \\
\text { aluno antes da simulação }\end{array}$ & - & - & - & - & 1 & 20 & 4 & 80 & 1,0 \\
\hline Descrição completa do cenário: script & - & - & - & - & 1 & 20 & 4 & 80 & 1,0 \\
\hline $\begin{array}{l}\text { Dados fornecidos ao aluno durante a simulação: } \\
\text { árvore de tomada de decisões }\end{array}$ & - & - & - & - & - & - & 4 & 80 & 1,0 \\
\hline Apoio fornecido ao aluno durante a simulação & - & - & - & - & 1 & 20 & 4 & 80 & 1,0 \\
\hline $\begin{array}{l}\text { Promoção da capacidade de priorizar avaliações e } \\
\text { intervenções de enfermagem }\end{array}$ & - & - & - & - & - & - & 4 & 80 & 1,0 \\
\hline Promoção da resolução autônoma de problemas & - & - & - & - & 1 & 20 & 4 & 80 & 1,0 \\
\hline $\begin{array}{l}\text { Adequação do quantitativo de atores/ papeis à } \\
\text { situação }\end{array}$ & - & - & - & - & - & - & 5 & 100 & 1,0 \\
\hline $\begin{array}{l}\text { Adequação do figurino e dispositivos utilizados pelos } \\
\text { atores em relação à simulação }\end{array}$ & - & - & - & - & - & - & 5 & 100 & 1,0 \\
\hline $\begin{array}{l}\text { Parâmetros do simulador/ atriz condizentes com o } \\
\text { caso clínico }\end{array}$ & - & - & - & - & - & - & 5 & 100 & 1,0 \\
\hline Ambiente simulado & - & - & - & - & 2 & 40 & 3 & 60 & 1,0 \\
\hline Materiais e equipamentos disponíveis aos alunos & - & - & - & - & 1 & 20 & 4 & 80 & 1,0 \\
\hline $\begin{array}{l}\text { Aspectos avaliados no debriefing } \\
\text { IVC Médio }\end{array}$ & - & - & - & - & - & - & 5 & 100 & $\begin{array}{r}1,0 \\
1,0\end{array}$ \\
\hline
\end{tabular}

Fonte: Elaborado pelas pesquisadoras a partir de dados obtidos do estudo. 
Durante o processo de validação, os juízes sugeriram pequenos ajustes. As sugestões contempladas foram: incluir o plano de parto como parte das documentações trazidas pela atriz junto com a Caderneta da Gestante; por conta das discordâncias entre OMS e Ministério da Saúde e pelo conhecimento obstétrico mais especializado, realizar o toque vaginal passou a ser uma conduta facultativa a depender da segurança do aluno em realizar este procedimento; tornou-se o acompanhante mais participativo da cena por meio de falas próprias; acrescentou-se na descrição sucinta que a gestante estava com fortes cólicas para elucidar o diagnóstico durante o cenário; modificaram-se os termos pelve feminina para simulador pélvico feminino para treinamento clínico avançado; e por último foi incluído na lista de materiais, um lençol para cobrir a maca do consultório.

\section{DISCUSSÃO}

O cenário proposto, por este estudo, considerou a realidade do Distrito Federal, por ser o local de atuação das pesquisadoras. Entretanto, com pequenas adaptações, ele pode ser utilizado em outras localidades, pois, atingir o realismo da cena em relação à característica local, é fundamental para provocar nos alunos as mesmas respostas psicológicas que teriam na prática clínica e produzir experiências de aprendizagem mais eficazes $^{(9)}$.

Visando a cumprir os objetivos de aprendizagem, é recomendado que o cenário seja conduzido por um facilitador experiente e que, se houver necessidade, sejam fornecidas dicas ao longo do cenário ${ }^{(17)}$. Para que haja uma padronização nessas dicas, foi elaborada a árvore de tomada de decisões. Esta fica em poder do facilitador para que ele vá controlando as dicas necessárias, conforme a atuação dos estudantes na situação. Além disso, o debriefing é fundamental, após o evento simulado para tornar o ambiente de aprendizagem apoiador, com comunicação aberta, e que busca promover nos alunos a autoanálise e reflexão sobre os vários aspectos abordados no cenário e sobre a criação de novas ações ${ }^{(7,18)}$. Para auxiliar na padronização do debriefing, desse cenário, foi validado também o checklist para a avaliação do ensino e aprendizado, que propõe não só critérios e objetivos a serem avaliados, durante a simulação, mas também perguntas que o facilitador pode se utilizar para estimular os estudantes a falarem nesse momento.

A etapa de validação é recomendada para confirmar a confiabilidade, segurança e aplicabilidade do cenário construído ${ }^{(14,11)}$. Destacase que a validação do cenário ocorreu de forma online, em uma única rodada de avaliação diante dos dados obtidos, com o IVC de 1,0 em todos os critérios avaliados, caso algum dos critérios tivesse obtido pontuação inferior, haveria uma nova rodada de avaliação.

O ponto de corte do IVC para se considerar um cenário validado é variável entre os estudos de validação, sendo que o índice de concordância aceitável entre os membros do comitê de especialistas deve ser de no mínimo 0,80 e, preferencialmente, maior que $0,90^{(19)}$. Sendo assim, os estudos recentemente publicados sobre validação de cenários clínicos, no contexto materno infantil e da saúde da mulher, seguiram esse padrão e consideraram parâmetros acima de 0,8 para o $\mathrm{IVC}^{(10,20-21)}$. Entretanto, o referencial adotado, neste estudo de validação propõe que, para estudos com corpo de juízes com menos de 6 profissionais, se considere como mínimo aceitável um IVC de 1,0 e esse valor reduz à medida que se amplia a quantidade de juízes ${ }^{(15)}$.

Apesar da obtenção de um IVC de 1,0, os juízes fizeram algumas sugestões. A maioria dessas sugestões foram acatadas e permitiram adequar o cenário e o instrumento de avaliação aos objetivos propostos, conferindo maior qualidade e realismo. Destaca-se que os estudos de validação de cenários no contexto da saúde da mulher publicados até o momento não propuseram cenários com essa mesma temática. Foram encontrados estudos com as seguintes temáticas: atendimento à gestante adolescente para diagnóstico de gravidez ${ }^{(20)}$, trabalho de parto e parto humanizados ${ }^{(21)}$ e manejo da hemorragia pós-parto(10). Assim, o cenário validado, neste estudo, consiste em uma nova temática, podendo impulsionar outras pesquisas sobre o tema.

É importante frisar que essa temática é de grande valia para a formação de futuros enfermeiros tendo em vista a autonomia e o papel fundamental da enfermagem, na assistência ao pré-natal de risco habitual na APS. Isso porque o enfermeiro deve ser qualificado para: identificar doenças ou intercorrências na gestação e intervir de forma resolutiva e precoce, prescrever medicamentos e solicitar exames estabelecidos em protocolos, identificar as necessidades primordiais da gestante, realizar um plano de assistência, 
oferecer as orientações essenciais, promover os encaminhamentos necessários e utilizar a humanização como base da sua prática assistencial ${ }^{(2,22)}$. Por isso, iniciativas que desenvolvam o pensamento crítico e a solução de problemas devem ser promovidas e encorajadas e a utilização das estratégias ativas de ensinoaprendizagem, como a simulação, podem aproximar o futuro enfermeiro das necessidades de saúde locais, regionais e nacionais ${ }^{(4)}$.

Estudo randomizado, realizado na China, contou com a participação de 177 acadêmicos de enfermagem e demonstrou que o grupo experimental que utilizou a metodologia de simulação como estratégia de ensino obteve um aumento significativo nas habilidades de julgamento crítico e clínico, em relação ao grupo que utilizou as metodologias tradicionais ${ }^{(23)}$. Outros estudos também evidenciaram que a simulação é uma estratégia capaz de agregar valor à educação superior em enfermagem e possui como pontos positivos a preparação dos alunos para a prática clínica, ao mesmo tempo em que favorece a correlação entre a teoria e prática (24).

Como pontos a serem melhorados na simulação como ferramenta de ensino, destacamse as questões emocionais como: medo, ansiedade e dificuldade de trabalhar em equipe no contexto da observação. Dessa forma, percebe-se a necessidade dos docentes de enfermagem elaborem estratégias e cenários que permitam minimizar esses fatores estressores, assim como foi proposto por esse estudo, de se tornar a etapa do debriefing um momento de aprendizagem e não de julgamento, dar dicas, ao longo do cenário, e ter um facilitador com experiência em ensino e simulação para mediar a simulação e suas etapas $(12,17,24)$

\section{CONCLUSÃO}

Conclui-se que o estudo alcançou os objetivos propostos, uma vez que o cenário e checklist de avaliação do ensino e aprendizagem validados obtiveram o valor máximo de IVC no processo de validação. As sugestões propostas pelas juízas resultaram em um cenário mais seguro e real para ser utilizado no ensino de enfermagem.

O estudo identificou uma lacuna no conhecimento acerca de validação de cenários para simulação clínica, no âmbito do atendimento pré-natal realizado pelo enfermeiro, portanto ressalta-se a importância de novos estudos sobre essa temática visando à ampliação do uso da simulação realística no ensino-aprendizado de saúde da mulher na formação de futuros enfermeiros.

A contribuição direta, deste estudo, para o ensino de enfermagem é incentivar a elaboração e validação de novos cenários e, indiretamente, ser um instrumento de aprimoramento do ensino em enfermagem, por estimular o uso da simulação nos cursos de graduação. A simulação tem sido amplamente utilizada e vem se mostrando um método eficaz, no preparo dos futuros enfermeiros para as situações clínicas reais, enfrentadas no cotidiano da profissão, dessa forma, entende-se que o estudo contribui para a formação de profissionais mais preparados no mercado de trabalho, especialmente na atenção pré-natal que exige raciocínio clínico e na tomada de decisões, por parte dos enfermeiros.

Considerou-se como limitações do estudo a ausência de estudos da mesma temática para comparativo de dados e a baixa adesão dos juízes para validação online do cenário, nesse contexto de pandemia.

\section{REFERÊNCIAS}

1 - Ministério da Saúde (BR). Atenção ao pré-natal de baixo risco. Brasília: Ministério da Saúde; 2012.

2 - Secretaria Estadual de Saúde do Distrito Federal. Portaria SES-DF no 342, de 28 de junho de 2017. Diário Oficial da União 2017.

3 - World Health Organization (WHO). WHO recommendations: Intrapartum care for a positive childbirth experience. Geneva: World Health Organization; 2018 [citado em 15 ago 2020]. Acesso

em: https://apps.who.int/iris/bitstream/handle/10665 /260178/9789241550215eng. pdf; jsessionid $=45 \mathrm{C} 4 \mathrm{DAEC} 668 \mathrm{C} 2 \mathrm{~B} 6 \mathrm{~A} 827 \mathrm{~A} 44 \mathrm{CO}$ 0518F4BF?sequence $=1$

4 - Felix AMS; Maia FOM; Soares RAQ. Atenção primária à saúde e educação em enfermagem no Brasil. Enferm Foco 2019;10(6):175-81. DOI: 10.21675/2357-707X.2019.v10.n6.2779

5 - Tjoflåt I, Våga BB, Søreide E. Implementing simulation in a nursing education programme: $\mathrm{A}$ case report from Tanzania. Adv Simul. 2017;2:17. DOI: $\underline{10.1186 / s 41077-017-0048-z}$

6 - Jerônimo IRL, Campos JF, Peixoto MAP, Brandão MAG. Use of clinical simulation to improve diagnostic reasoning in nursing. Esc Anna Nery 
2018;22(3):1-9. DOI: $10.1590 / 2177-9465-e a n-$ $\underline{2017-0442}$

7 - International Nursing Association for Clinical Simulation and Learning. INACSL standards of best practice: Simulation ${ }^{\mathrm{SM}}$ Debriefing. Clin Simul Nurs. 2016a;12(Suppl):S21-5.

DOI: 10.1016/j.ecns.2016.09.008

8 - Negri EC, Pereira JGA, Cotta FCK, Franzon JC, Mazzo A. Construction and validation of simulated scenario for nursing care to colostomy patients. Texto Context Enferm. 2019;28:1-16. DOI: $\underline{10.1590 / 1980-265 x-t c e-2018-0199}$

9 - Brady S, Bogossian F, Gibbons K. The effectiveness of varied levels of simulation fidelity on integrated performance of technical skills in midwifery students-A randomised intervention trial. Nurse Educ Today 2015; 35(3):524-9. DOI: 10.1016/i.nedt.2014.11.005

10 - Andrade PON, Oliveira SC, Morais SCRV, Guedes TG, Melo GP, Linhares FMP. Validação de cenário de simulação clínica no manejo da hemorragia pós-parto. Rev Bras Enferm. 2019;72(3):624-31. DOI: 10.1590/0034-71672018-0065

11 - Fabri RP, Mazzo A, Martins JCA, Fonseca AS, Pedersoli CE, Miranda FBG, et al. Development of a theoretical-practical script for clinical simulation. Rev Esc Enferm USP 2017;51:e03218. DOI: 10.1590/s1980-220×2016265103218.

12 - Rodrigues FL, Moura LM, Boeckmann LMM, Melo MC, Vasconcelos FC, SantAna G. Avaliação do processo ensino e aprendizagem no ambiente de simulação realística na graduação em enfermagem. Enferm Foco 2019; 10(6):118-24. DOI: $\underline{10.21675 / 2357-707 X .2019 . v 10 . n 6.2782}$

13 - Gilbert M, Adamson KA, Bodily D, Stauffenecker $C$, Ingram K, Guerne A, et al. Making Sense of Methods and Measurement: Validation part II. Clin Simul Nurs. 2016;12:275-6. DOI: $\underline{10.1016 / j . e c n s .2016 .02 .006}$

14 - Góes FSN, Dalri MCB, Fonseca LMM, Canini SEMS, Scochi CGS. Desenvolvimento de casos clínicos para o ensino do raciocínio diagnóstico. Rev Eletrônica Enferm. 2014;16(1):441. DOI: $\underline{10.5216 / \text { ree.v16i1.19812 }}$

15 - Alexandre NMC, Coluci MZO. Content validity in the development and adaptation processes of measurement instruments. Ciênc Saúde Coletiva 2011;16(7):3061-8. DOI: 10.1590/S1413$\underline{81232011000800006}$

16 - Conselho Nacional de Saúde. Resolução no 466, de 12 de dezembro de 2012. Diário Oficial da União 2013;12(1):59.

17 - International Nursing Association for Clinical Simulation and Learning. INACSL standards of best practice: SimulationSM facilitation. Clin Simul Nurs. 2016b;12(Suppl):S16-S20. DOI: j.ecns.2016.09.007

18 - Presado MHCV, Colaço S, Rafael H, Baixinho $\mathrm{CL}$, Félix I, Saraiva $\mathrm{C}$, et al. Aprender com a Simulação de Alta Fidelidade. Ciênc Saúde Coletiva 2018;23(1):51-9. DOI: 10.1590/1413$\underline{81232018231.23072017}$

19 - Souza AC, Alexandre NMC, Guirardello EB. Propriedades psicométricas na avaliação de instrumentos: Avaliação da confiabilidade e da validade. Epidemiol Serv Saúde 2017;26(3):649-59. DOI: $10.5123 / s 1679-49742017000300022$

20 - Leon CGRMP, Silva AK, Ribeiro LM, Brasil GC, Guarda LEA, Fonseca LMM. Construção e validação de casos clínicos para utilização no ensino de enfermagem no contexto materno-infantil. Rev Enf Ref. 2018;4(18):51-62. DOI: 10.12707/RIV1801

21 - Fonseca LMM, Monteiro JCS, Aredes NDA, Bueno JV, Domingues AN, Coutinho VRD, et al. Interdisciplinary simulation scenario in nursing education: Humanized childbirth and birth. Rev Latino-Am Enfermagem 2020;28:1-10. DOI: $\underline{10.1590 / 1518-8345.3681 .3286}$

22 - Gomes CBA, Dias RS, Silva WGB, Pacheco $M A B$, Sousa FGM, Loyola CMD. Prenatal nursing consultation: Narratives of pregnant women and nurses. Texto Contexto-Enferm. 2019;28:1-15. DOI: 10.1590/1980-265x-tce-2017-0544

23 - Yang F, Wang Y, Yang C, Zhou MH, Shu J, Fu B, et al. Improving clinical judgment by simulation: a randomized trial and validation of the Lasater clinical judgment rubric in Chinese. BMC Med Educ. 2019;19(1):20. DOI: 10.1186/s12909-019-1454-9

24 - Rosa MEC, Pereira-Ávila FMV, Góes FGB, Pereira-Caldeira NMV, Sousa LRM, Goulart MCL. Positive and negative aspects of clinical simulation in nursing teaching. Esc Anna Nery 2020;24(3):1-9. DOI: 10.1590/2177-9465-ean-2019-0353 
Portela RG, Viduedo AFS, Ribeiro LM, et al. 11

Nota: Este estudo faz parte da monografia de conclusão de curso da primeira autora.

Recebido em: 01/12/2020

Aprovado em: 07/02/2021 